\title{
Technical Note: Novel method for water vapour monitoring using wireless communication networks measurements
}

\author{
N. David ${ }^{1}$, P. Alpert ${ }^{1}$, and H. Messer ${ }^{2}$ \\ ${ }^{1}$ The Department of Geophysics and Planetary Sciences, Tel-Aviv University, Tel-Aviv, Israel \\ ${ }^{2}$ The School of Electrical Engineering, Tel-Aviv University, Tel-Aviv, Israel
}

Received: 22 April 2008 - Published in Atmos. Chem. Phys. Discuss.: 12 June 2008

Revised: 16 March 2009 - Accepted: 25 March 2009 - Published: 3 April 2009

\begin{abstract}
We propose a new technique that overcomes the obstacles of the existing methods for monitoring near-surface water vapour, by estimating humidity from data collected through existing wireless communication networks.

Weather conditions and atmospheric phenomena affect the electromagnetic channel, causing attenuations to the radio signals. Thus, wireless communication networks are in effect built-in environmental monitoring facilities. The wireless microwave links, used in these networks, are widely deployed by cellular providers for backhaul communication between base stations, a few tens of meters above ground level. As a result, if all available measurements are used, the proposed method can provide moisture observations with high spatial resolution and potentially high temporal resolution. Further, the implementation cost is minimal, since the data used are already collected and saved by the cellular operators. In addition - many of these links are installed in areas where access is difficult such as orographic terrain and complex topography. As such, our method enables measurements in places that have been hard to measure in the past, or have never been measured before. The technique is restricted to weather conditions which exclude rain, fog or clouds along the propagation path. Strong winds that may cause movement of the link transmitter or receiver (or both) may also interfere with the ability to conduct accurate measurements.

We present results from real-data measurements taken from two microwave links used in a backhaul cellular network that show convincing correlation to surface station humidity measurements. The measurements were taken daily in two sites, one in northern Israel (28 measurements), the other
\end{abstract}

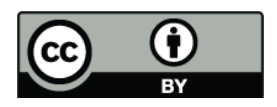

Correspondence to: N. David (noamda@post.tau.ac.il) in central Israel (29 measurements). The correlation between the microwave link measurements and the humidity gauges were 0.9 and 0.82 for the north and central sites, respectively. The Root Mean Square Differences (RMSD) were $1.8 \mathrm{~g} / \mathrm{m}^{3}$ and $3.4 \mathrm{~g} / \mathrm{m}^{3}$ for the northern and central site measurements, respectively.

\section{Introduction}

Atmospheric humidity has a cardinal part in a variety of environmental processes (e.g. Allan et al., 1999) in many fields. As the most influential of greenhouse gases, it absorbs longwave terrestrial radiation. The water vapour cycle of evaporation and recondensation is a major energy redistributing mechanism transferring heat energy from the Earth's surface to the atmosphere. Meteorological decision-support for weather forecasting is based on atmospheric model results (e.g. Shay-El and Alpert, 1991), the accuracy of which is determined by the quality of its initial conditions or forcing data. Hence, humidity, in particular, is a crucial variable for the initialization of atmospheric models. One of the central conclusions of the Mesoscale Alpine Programme (MAP), aimed at improving prediction of the regional weather and particularly rainfall and flooding, was that accurate moisture fields for initialization are essential (Ducrocq et al., 2002).

Current methods for obtaining humidity measurements include predominantly: surface stations, radiosondes and satellite systems. Common humidity instruments, found in surface stations, suffer from low spatial resolution since they provide only very local point observations. Moisture, in particular, is a field having unusually high variability in the mesoscale as demonstrated, for instance, by structure

Published by Copernicus Publications on behalf of the European Geosciences Union. 
functions (Lilly and Gal-Chen, 1983). Furthermore, over heterogeneous terrain and complex topography, the spread of gauges is even more restricted due to often poor accessibility and positioning difficulties. Satellites, although they cover large areas, are frequently not accurate enough at surface levels while it is the near-surface moisture level that is, in most cases, the crucial variable for convection. Radiosondes, which are typically launched only $2-4$ times a day, also provide very limited information. Additionally, these monitoring methods are costly for implementation, deployment and maintenance.

For model initialization, a point moisture measurement close to the surface (about $2 \mathrm{~m}$, as in a standard meteorological station) is not satisfactory due to local surface perturbations. For meteorological modeling purposes, an area average representing the near-surface moisture at an altitude of a few tens of meters, over a box with the scale of the model's grid, is required. This type of data cannot, with use of current measuring tools, be effectively collected. The method we present provides a unique way of obtaining it.

As weather conditions and atmospheric phenomena cause impairments on radio links, wireless communication networks provide built-in environmental monitoring tools, as was recently demonstrated for rainfall (Messer et al., 2006, 2007; Leijnse et al., 2007) and areal evaporation (Leijnse et al., 2007) observations. In this paper we introduce a new technique to measure atmospheric humidity using data collected by wireless systems. Wireless communication, and in particular cellular networks, are widely distributed, operating in real time with minimum supervision, and therefore can be considered as continuous, high resolution humidity observation apparatus.

Environmental monitoring using data from wireless communication networks offers a completely new approach to quantifying ground level humidity. Since cellular networks already exist over large regions of the land, including complex topography such as steep slopes and since the method only requires standard data (saved by the communication system anyway), the costs are minimal.

Of the various wireless communication systems, we focus on the microwave point-to-point links which are used for backhaul communication in cellular networks, as they seem to have the most suitable properties for our purposes: they are static, line-of-sight links, built close to the ground, and operate in a frequency range of tens of $\mathrm{GHz}$.

In this research, the wireless system used for humidity observations has a magnitude resolution of $0.1 \mathrm{~dB}$ per link. This communication network provides attenuation data every few seconds, but only stores one datum point per $24 \mathrm{~h}$ (at 03:00 a.m.).The system can be configured to store data at shorter time intervals, it is a matter of technical definition by the cellular companies. Therefore, it has the potential of providing moisture observations at high temporal resolution. The length of an average microwave link is on the order of a few $\mathrm{km}$ and tends to be shorter in urban areas and longer in rural regions. In typical conditions of $1013 \mathrm{hPa}$ pressure, $15^{\circ} \mathrm{C}$ temperature and water vapour density of $7.5 \mathrm{~g} / \mathrm{m}^{3}$, the attenuation caused to a microwave beam interacting with the water vapour molecules at a frequency of $\sim 22 \mathrm{GHz}$ is roughly around $0.2 \mathrm{~dB} / \mathrm{km}$ (Rec. ITU-R P.6766, 2005; Liebe, 1985). Therefore, perturbations caused by humidity can be detected. Rain, fog and clouds create additional attenuation in relation to that caused by water vapour. One of the research challenges we are faced with is separating the effects of different attenuation sources. As we aim to prove feasibility, at this stage, the technique is limited to periods where none of the aforementioned phenomena exist along the link line-of-sight. The microwave links are sensitive to mechanical oscillations. Therefore, strong winds, that may cause movement of either the receiver or the transmitter (or both), may also be considered as a source of error (Leijnse et al., 2007).

Another point of interest is the signal delay (and its variations) of the communication links studied. Using this delay provides an advantage in that in practice it is not affected by fog, clouds or rain along the propagation path. This fact is used in the application of inferring the atmospheric water vapour content from the signals of the GPS satellites (Bevis et al., 1992). However, the data presently used do not provide time delay values.

\section{Theory and methods}

At frequencies of tens of $\mathrm{GHz}$, the main absorbing gases in the lower atmosphere are oxygen and water vapour. While oxygen has an absorption band around $60 \mathrm{GHz}$, water vapour has a resonance line at $22.235 \mathrm{GHz}$. Although other atmospheric molecules have spectral lines in this frequency region, their expected strength is too small to affect propagation significantly (Raghavan, 2003; Meeks, 1976).

As a consequence, an incident microwave signal, interacting with an $\mathrm{H}_{2} \mathrm{O}$ molecule, might be attenuated, specifically if its frequency is close to the molecule's resonant one. Since backhaul links in cellular networks often operate around frequencies of 22 to $23 \mathrm{GHz}$, we focus on the $22.235 \mathrm{GHz}$ absorbing line to monitor the water vapour.

The specific attenuation $\gamma[\mathrm{dB} / \mathrm{km}]$ due to dry air and water vapour, at centimeter wavelengths, is well studied and can be evaluated (Rec. ITU-R P.676-6, 2005; Liebe, 1985) using the following procedure:

$$
\begin{aligned}
& \gamma=A_{W}+A_{0} \quad[\mathrm{~dB} / \mathrm{km}] \\
& \gamma=\frac{4 \pi f N^{\prime \prime}}{c}\left[\mathrm{~m}^{-1}\right]=0.1820 f N^{\prime \prime}[\mathrm{dB} / \mathrm{km}]
\end{aligned}
$$

Where:

$A_{w}$ : The specific attenuation due to water vapour $[\mathrm{dB} / \mathrm{km}]$.

$A_{o}$ : The specific attenuation due to dry air $[\mathrm{dB} / \mathrm{km}]$ (Assuming the air is moist, $A_{o}$ is one order of magnitude lower 
than $A_{w}$ since at frequencies of $\sim 22 \mathrm{GHz}$, the attenuation is caused predominantly by the water vapour).

$f$ : The link's frequency [GHz].

$N "=N ”(p, T, \rho, f)$ : The imaginary part of the complex refractivity, measured in $\mathrm{N}$-units, a function of the pressure $p[\mathrm{hPa}]$, temperature $T\left[{ }^{\circ} \mathrm{C}\right]$, frequency $f[\mathrm{GHz}]$ and the water vapour density $\rho\left[\mathrm{g} / \mathrm{m}^{3}\right]$.

While:

$N^{\prime \prime}=\sum_{i} S_{i} F_{i}+N_{D}^{\prime \prime}$

$S_{i}=S_{i}(p, T, \rho, f)$ : The strength of the $i$-th line [KHz].

$F_{i}=F_{i}(p, T, \rho, f)$ : Line shape factor $\left[\mathrm{GHz}^{-1}\right]$.

$N{ }^{\prime}{ }_{D}=N{ }^{\prime}{ }_{D}(p, T, f)$ : The dry continuum due to pressureinduced nitrogen absorption and the Debye spectrum.

The summation is of the individual resonance lines from oxygen and water vapour, the sum extends over all lines up to $1000 \mathrm{GHz}$.

The detailed expression of the functions of $N^{\prime \prime}$ is described in the literature (Rec. ITU-R P.676-6, 2005; Liebe, 1985).

Given measurements of the Received Signal Level (RSL), $\gamma$ can be derived based on the microwave link's measurements.

The RSL value chosen as the point of reference, only needs to be set once for each link, by subtracting the attenuation created by a typical moist air in the area over several weeks from the median RSL measurement of these weeks.

Consequently, given the atmospheric temperature, pressure and the link's frequency, the water vapour density $\rho$ $\left[\mathrm{g} / \mathrm{m}^{3}\right]$ is estimated numerically through Eq. (2), using the known relation between $N^{\prime \prime}$ and $\rho$.

As meteorological surface stations normally do not provide the absolute moisture $\rho$, it was derived using the following formulas (Rec. ITU-R P.676-6, 2005; Liebe, 1985; Bolton, 1980):

$$
\begin{aligned}
& e_{s}=6.112 \exp \left(\frac{17.67 T}{T+243.5}\right) \\
& e=\rho \frac{T+273.15}{216.7} \\
& \frac{e}{e_{s}} 100 \% \equiv \mathrm{RH}
\end{aligned}
$$

$e_{s}-$ The saturation water vapour pressure $[\mathrm{hPa}]$.

$e$ - The water vapour partial pressure $[\mathrm{hPa}]$.

$T$ - The temperature $\left[{ }^{\circ} \mathrm{C}\right]$.

$\rho-$ The water vapour density $\left[\mathrm{g} / \mathrm{m}^{3}\right]$.

Hence:

$\rho=1324.45 \times \frac{\mathrm{RH}}{100 \%} \times \frac{\exp \left(\frac{17.67 T}{T+243.5}\right)}{T+273.15}$

\section{Statistical tests}

We investigated the correlation between absolute humidity values calculated using the method described, and those which were measured using a regular humidity gauge. The correlation analysis was performed by the Pearson's correlation test, while the level of significance was set to 0.05 . P-values were also calculated (Neter et al., 1996).

The Root Mean Square Difference (RMSD) was used according to the following definition:

$\operatorname{RMSD}\left[\mathrm{g} / \mathrm{m}^{3}\right]=\sqrt{\frac{\sum_{i=1}^{N}\left(\rho_{m i}-\rho_{g i}\right)^{2}}{N}}$

$\rho_{m i}$ - The $i$-th water vapour density measurement as measured using the microwave link $\left[\mathrm{g} / \mathrm{m}^{3}\right]$.

$\rho_{g i}-$ The $i$-th water vapour density measurement as measured using the humidity gauge $\left[\mathrm{g} / \mathrm{m}^{3}\right]$.

$N$ - The number of samples (28 samples were taken from the northern site and 29 from the central Israel site). The humidity measurements taken via the microwave link were calculated from a signal instantaneously sampled at 03:00 a.m. Humidity measurements with the regular humidity gauge were taken at the surface stations every half hour, and from these measurements, the ones relating to the same hour were selected.

\section{Results}

Moisture observations using microwave links were made in several different locations in Israel, and at several different times. The results presented here (Figs. 1 and 2) are for Haifa (northern Israel, link frequency $22.725 \mathrm{GHz}$ ) and Ramla (central Israel, 21.325 GHz), during November 2005 and April-May 2007, respectively.

Figure 2 presents results for inter-daily variations in the absolute moisture which were calculated using data obtained from the wireless communication network, as compared to in-situ measurements, over a month. The results show a persuasive match between the conventional technique and the novel method, the correlation coefficient between the time series in the two presented cases is 0.9 and 0.82 , respectively. In both cases, the $p$ value is less than 0.05 . The RMSD were found to be $1.8 \mathrm{~g} / \mathrm{m}^{3}$ for the northern site and $3.4 \mathrm{~g} / \mathrm{m}^{3}$ for the central site measurements. The presented results (2 cases) are examples of other cases, all demonstrating relatively good agreements. Similar comparisons were performed for other links and other time slots showing correlations in the range of $0.5-0.9$. The system from which the data were collected captures a single signal every $24 \mathrm{~h}$ at 03:00 a.m. The surface station observations used were taken from the vicinity of the link's area at the same hour. Since rainfall causes additional signal-attenuation, days when showers occurred 


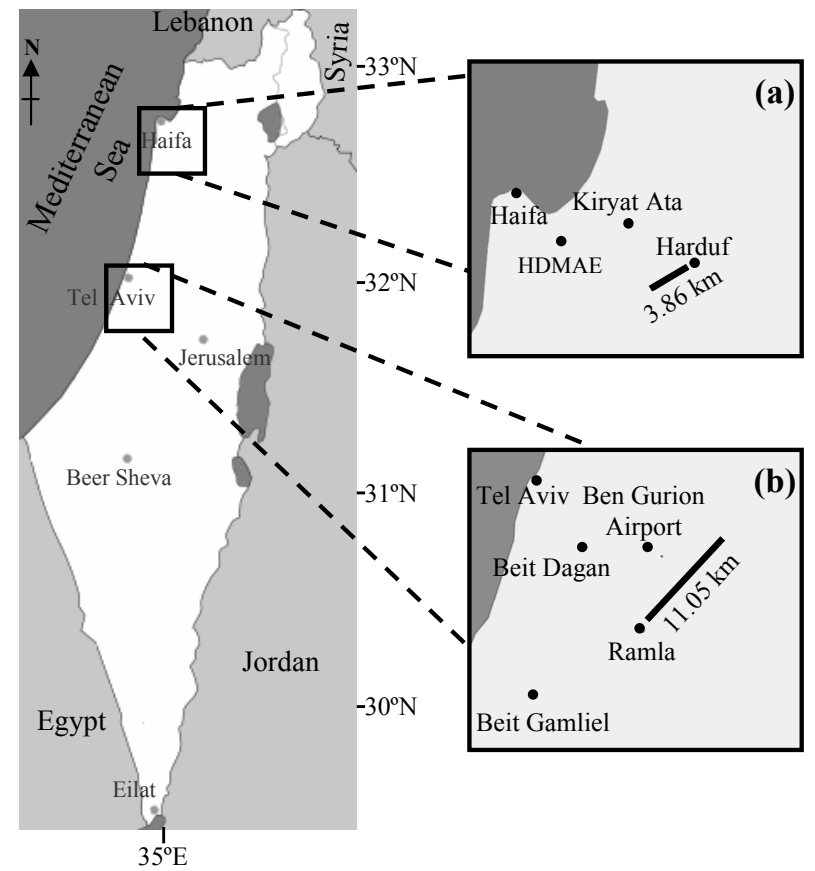

Fig. 1. The examined regions:

(a) The microwave link ( $3.86 \mathrm{~km}$ long, marked as a line) in front of Kiryat Ata (where the humidity gauge is located), Haifa bay. The distance from the surface station to a point located in the middle of the wireless link is $7.5 \mathrm{~km}$. The surface station is situated $45 \mathrm{~m}$ a.s.1., while the microwave transmitter and receiver are located on two hills: 265 and $233 \mathrm{~m}$ a.s.1.

(b) The microwave link $(11.05 \mathrm{~km})$ in front of Ben-Gurion airport (humidity gauge's location), central Israel. The distance from the surface station to a point located in the middle of the link is $5 \mathrm{~km}$. The airport surface station is situated at $41 \mathrm{~m}$ a.s.l., while the link's transmitter and receiver are located at heights of 116 and $98 \mathrm{~m}$ a.s.l.

approximately at 03:00 a.m. till 04:00 a.m. (according to close by surface stations), were excluded.

The largest difference between the traditional and the novel measurement methods (Fig. 2b) appears on the night of 6 May 2007. This night was a holiday in Israel (Lag Ba'omer), where hundreds of bonfires were lit all across the country. As a result, many particles were released into the low atmosphere speeding up the creation of smog and possibly fog (the measured relative humidity by a radiosonde launched at 03:00 a.m. from Beit Dagan (Fig. 1b), a few km away from the microwave link, at an altitude of $95 \mathrm{~m}$ Above Sea Level (a.s.1.) was 97\%). The reason for the additional attenuation observed by the microwave link (expressed by a higher moisture level) might be due to local fog (Raghavan, 2003), implying that the system may provide the ability to monitor this phenomenon through the use of wireless communication data. When excluding the 6 May measurement, the correlation increases to 0.85 and the RMSD decreases to $2.9 \mathrm{~g} / \mathrm{m}^{3}$. Further investigation is needed concerning this point.
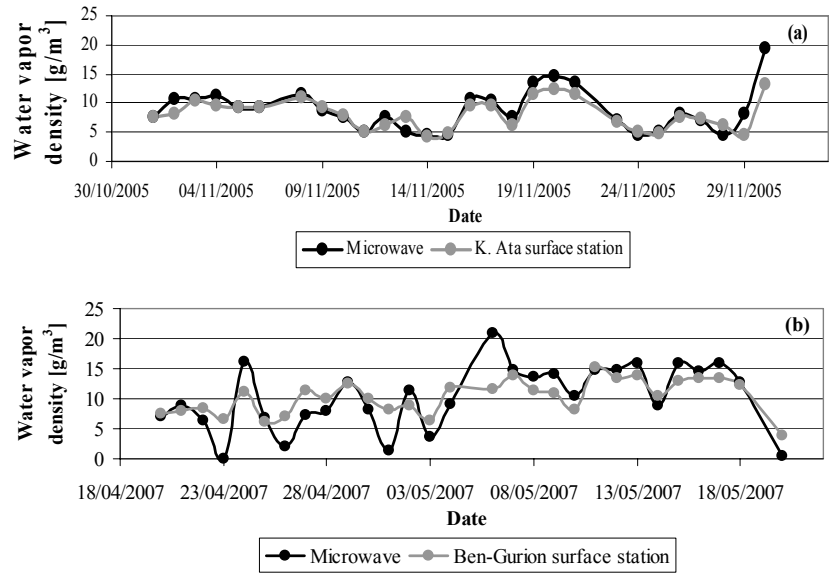

Fig. 2. The water vapour density $\rho\left[\mathrm{g} / \mathrm{m}^{3}\right]$ as estimated using RSL measurements from the microwave link data (dark) vs. conventional humidity gauge data (bright).

Northern Israel - The observations were made during the month of November 2005, where 2 rainy days were excluded ( 7 and 22 November). The rainfall data were taken from two different surface stations situated in the Haifa District Municipal Association for the Environment (HDMAE) and in Kiryat Ata, about $12.5 \mathrm{~km}$ and 7 $\mathrm{km}$, respectively, from Harduf (see Fig. 1a). The link's frequency is $22.725 \mathrm{GHz}$. The calculated correlation between the two curves is 0.9 while the RMSD is $1.8 \mathrm{~g} / \mathrm{m}^{3}$.

Central Israel - The measurements were taken between 20 April and 20 May 2007, excluding 2 days when showers occurred (5 and 19 May). The precipitation data were taken from Beit Gamliel surface station which is located about $13 \mathrm{~km}$ from Ramla (see Fig. 1b). It should be noted that it is possible that the increased attenuation in this case that is greater than the typical moist air attenuation, was caused as a result of other interference such as wind moving the transmitter or receiver (Leijnse et al., 2007). As there was a surface station that recorded precipitation in the area, the increased attenuation was ascribed to precipitation. Further investigation is required to identify the sources of these perturbations. The link's frequency is $21.325 \mathrm{GHz}$ and the calculated correlation between the time series is 0.82 with RMSD of $3.4 \mathrm{~g} / \mathrm{m}^{3}$.

\section{Uncertainties}

The following uncertainties were calculated based on the model (Rec. ITU-R P.676-6, 2005; Liebe, 1985) used in this study. The dominant uncertainty affecting the absolute humidity calculation is that of the attenuation quantization error. Due to $0.1 \mathrm{~dB}$ quantization error per link, the uncertainty in evaluating attenuation, for a $3.86 \mathrm{~km}$ link, is \pm 0.026 $\mathrm{dB} / \mathrm{km}$. As a result we get that the error in calculating absolute humidity for this link is of the magnitude of $\pm 1 \mathrm{~g} / \mathrm{m}^{3}$. In the case of an $11.05 \mathrm{~km}$ link, the uncertainty in evaluating the attenuation is $\pm 0.01 \mathrm{~dB} / \mathrm{km}$, hence the corresponding error in calculating the absolute humidity is of the magnitude of $\pm 0.5 \mathrm{~g} / \mathrm{m}^{3}$. Dry air effect on attenuation is one order of magnitude lower than that of water vapour in this case. Quantitatively it is $0.01 \mathrm{~dB} / \mathrm{km}$ for dry air and $0.19 \mathrm{~dB} / \mathrm{km}$ 
as a result of humidity (for a link operating near $22 \mathrm{GHz}$, temperature of $15^{\circ} \mathrm{C}$, humidity of $7.5 \mathrm{~g} / \mathrm{m}^{3}$ and a sea level pressure). However, the algorithm takes into account the effects of dry air, and corrects for them. Another atmospheric parameter which can be estimated based on the model is the imaginary part of the refractive index- $N^{\prime \prime}$, this variable represents the absorption. Under the same atmospheric conditions as mentioned previously and for a link operating near $22 \mathrm{GHz}$, a typical value which was obtained for this variable, based on the model, is: $0.045 \mathrm{~N}$-units. The uncertainty depends on the path length, it is: $\pm 0.006 \mathrm{~N}$-units and \pm 0.003 $\mathrm{N}$-units for the $3.86 \mathrm{~km}$ and the $11.05 \mathrm{~km}$ links, respectively.

The uncertainties in measuring temperature and pressure are of the magnitude 0.1 degrees Celsius, and $1 \mathrm{mb}$, respectively. But changes of this magnitude in pressure or temperature do not create a significant change in the absolute humidity calculation based on this model.

The estimated uncertainty in measuring humidity with regular humidity gauges is about 0.2 to $0.5 \mathrm{~g} / \mathrm{m}^{3}$ (depending on the relative humidity and the temperature), while the error in measuring relative humidity was taken to be $3 \%$.

\section{Conclusions}

The calculated, theoretical uncertainty for the measurement of the Ramla (central Israel) link is smaller in relation to the calculated uncertainty for the Harduf (northern Israel) link. The measurements from the northern link, on the other hand, present a better correspondence with the humidity gauge readings, which seems to go against the expectations from the error calculations. While it will need to be further studied, we can suggest several reasons for the observed discrepancy:

It is important to note that the transmitting and receiving units of the Harduf link are located on hilltops, and are higher off the ground, so that the microwave beam travels over a valley. On the other hand, while the Ramla link is located some $100 \mathrm{~m}$ a.s.l. its transmitter and receiver are only 18 and $27 \mathrm{~m}$ above the surface of a fairly flat plane. It is possible then, that the Ramla link is more prone to surface reflection and interference (Leijnse et al., 2007).

The representativeness of the spot humidity gauges is also an error factor. It is possible that the humidity gauge in the Harduf area better represents the average humidity in the area than the Ramla humidity gauge does. Thus, it is possible that, while the distance between the humidity gauge and microwave link in the Ramla area is smaller than the distance in the Harduf region, the measurements of the Ramla humidity gauge do not correspond as well to the measurements of the microwave link that represent the average humidity along the link (a distance of some $11 \mathrm{~km}$ ). Furthermore, the difference in location between the measurement sites and particularly the difference in the moisture level with altitude which can be significant at night hours (especially if an inversion layer is being crossed), introduces additional disparities between the microwave measurements and those made by the conventional humidity gauges.

Given the newly available data provided by the wireless communication facilities, improved initialization of atmospheric models can be achieved, thus enhancing prediction and hazards warning skills as well as providing a better understanding of the global climate system.

Acknowledgements. We wish to acknowledge and thank Y. Dagan and Y. Eisenberg (Cellcom) for their cooperation and for providing the microwave data.

We also thank B. Goldman (Haifa District Municipal Association for the Environment) and A. Arie (Meteo-tech) for humidity gauge data.

In addition, we would like to thank our research team members: A. Zinevich, A. Rayitsfeld and U. Hadar (Tel Aviv University) for their advice and assistance throughout the research.

Furthermore, many thanks to the reviewers whose comments we have found to be tremendously helpful.

This work was supported by a grant from the Yeshaya Horowitz Association, Jerusalem.

Additional support was given by the PROCEMA-BMBF project and by the GLOWA-JR BMBF project.

Edited by: S. Buehler

\section{References}

Allan, R. P., Shine, K. P., Slingo, A., and Pamment, J. A.: The dependence of clear-sky outgoing long-wave radiation on surface temperature and relative humidity, Q. J. Roy. Meteor. Soc., 125, 2103-2126, 1999.

Bevis, M., Businger S., Herring, T. A., Rocken, C., Anthes, R. A., and Ware, R. H.: GPS meteorology remote sensing of atmospheric water vapor using the global positioning system, J. Geophys. Res., 97, 15787-15801, 1992.

Bolton, D.: The computation of equivalent potential temperature, Mon. Weather Rev., 108, 1046-1053, 1980.

Ducrocq, V., Ricard, D., Lafore, J. P., and Orain, F.: Storm-scale numerical rainfall prediction for five precipitating events over France: On the importance of the initial humidity field, Weather Forecast, 17, 1236-1256, 2002.

Leijnse, H., Uijlenhoet, R., and Stricker, J. N. M.: Rainfall measurement using radio links from cellular communication networks, Water Resour. Res., 43, W03201, doi:10.1029/2006WR005631 ,2007.

Leijnse, H., Uijlenhoet, R., and Stricker, J. N. M.: Hydrometeorological application of a microwave link: 1. Evaporation, Water Resour. Res., 43, W04416, doi:10.1029/2006WR004988, 2007.

Leijnse, H., Uijlenhoet, R., and Stricker, J. N. M.: Hydrometeorological application of a microwave link: 2. Precipitation, Water Resour. Res., 43, W04417, doi:10.1029/2006WR004989, 2007.

Liebe, H. J.: An updated model for millimeter wave propagation in moist air, Radio Sci., 20, 1069-1089, 1985.

Lilly, D. K. and Gal-Chen, T.: North Atlantic Treaty Organization \& Scientific Affairs Division. Mesoscale meteorology-theories, 
observations, and models, Reidel, Dordrecht, The Netherlands, 13-24, 1983.

Meeks, M. L.: Astrophysics, Academic Press. New York, 142-176, 1976.

Messer, H., Zinevich, A., and Alpert, P.: Environmental monitoring by wireless communication networks, Science, 312, 713, 2006.

Messer, H.: Rainfall monitoring using cellular networks, IEEE Signal Proc. Mag., 24, 142-144, 2007.

Neter, J., Kutner, M. H., Nachtsheim, C., and Wasserman, W.: Applied Linear Statistical Models, 4th Edition, McGraw Hill, Inc., 640-645, 1996.
Raghavan, S.: Radar Meteorology. Kluwer Academic Publishers, Dordrecht The Netherlands, 51-91, 2003.

Rec. ITU-R P.676-6: Attenuation by atmospheric gases, ITU-R Recommendations, September 2005.

Shay-El, Y. and Alpert, P.: A diagnostic study of winter diabatic heating in the Mediterranean in relation to cyclones, Q. J. Roy. Meteor. Soc., 117, 715-747, 1991. 